\title{
Kinetics of magnetic flocculation. I: Flocculation of colloidal particles
}

\author{
J J M Janssen, J J M Baltussen, A P van Gelder and \\ J A A J Perenboom
}

High Field Magnet Laboratory and Research Institute for Materials, University of Nijmegen, Toernooiveld, NL-6525 ED Nijmegen, The Netherlands

Received 1 August 1989, in final form 30 July 1990

\begin{abstract}
The magnetic dipole-dipole interaction between magnetized colloidal particles can lead to particle aggregation (flocculation), when it is strong enough to outweigh the stabilizing forces of electro-chemical origin. An understanding of the timescale at which the magnetic flocculation process is developing is of essential importance for its practical use as a separation technique. Incorporation of the magnetic dipole-dipole interaction between the particles into the well established Fuchs theory of flocculation kinetics is difficult, due to the fact that this magnetic interaction is not spherically symmetric. In this paper a numerical approach is presented, in which the basic equation for flocculation is solved allowing for a cylindrically symmetric interaction, such as due to the presence of induced magnetic moments in paramagnetic particles. Experimental observations on the flocculation kinetics of fine-particle (about $0.2 \mu \mathrm{m}$ diameter) electrostatically stabilized $\mathrm{Mn}_{2} \mathrm{O}_{3}$ colloids corroborate the predictions of our model calculations.
\end{abstract}

\section{Introduction}

Colloidal solutions, liquids containing sub- $\mu$ m-sized particles, play an important role in many natural phenomena as well as in various industrial processes. In all cases the stability of these systems against aggregation (flocculation) of the particles is of essential importance for their behaviour. This stability is determined by the interplay between the particle's Brownian motion and several interparticle interactions, as described by the well established Derjaguin-LandauVerwey-Overbeek (DLVo) theory $[1,2]$. When a colloid is unstable, the timescale of the flocculation process is of both fundamental and practical importance. In colloid chemistry [1, 2] and aerosol science [3] flocculation kinetics has been a subject of interest for almost a century. The first mathematical model of flocculation kinetics has been given by von Smoluchowski [4] in 1917, for a colloid in which only the van der Waals attraction is present. A model for socalled slow flocculation, which allows incorporation of the electric double layer repulsion, was formulated in 1934 by Fuchs [5]. This double-layer interaction results from the fact that colloidal particles in solution are usually charged, due to adsorption of certain ions from the liquid, or to dissociation of polar surface groups $[1,2]$. Extensions and refinements of these models, concerning among others the polydispersity of real systems and the possibility of reversible flocculation, have been presented in later years [2]. In all cases, the interactions considered were spherically symmetric.
When a colloid is exposed to an applied magnetic field, the magnetic interaction between the permanent or induced magnetic moments has to be taken into account. This interaction however, is not spherically symmetric, but depends on the angle between the interacting magnetic dipole moments $\boldsymbol{m}_{1}$ and $\boldsymbol{m}_{2}$ :

$$
V_{\mathrm{M}}=\frac{\mu_{0}}{4 \pi} \frac{\boldsymbol{m}_{1} \cdot \boldsymbol{m}_{2}-3\left(\boldsymbol{m}_{1} \cdot \hat{\boldsymbol{r}}\right)\left(\boldsymbol{m}_{2} \cdot \hat{\boldsymbol{r}}\right)}{r^{3}}
$$

where $r=r \hat{r}$ is the vector connecting the particle centres, and $\mu_{0}$ the permeability of free space. For a head-tail configuration of these moments the magnetic interaction is attractive. When this attraction is strong enough to outweigh the stabilizing forces, magnetically induced aggregation (usually called magnetic flocculation) occurs [6]. This phenomenon can be used in practice as a separation technique, e.g. by choosing the field and other circumstances such that only one component of a mixture flocculates and settles.

Only few authors have discussed the kinetics of magnetic flocculation up to now. Svoboda [6] includes the magnetic interaction directly into the results of the colloid-chemical models, approximating this interaction for all angles by its value for maximum attraction (i.e. $\boldsymbol{m}_{1}$ and $\boldsymbol{m}_{2}$ parallel in equation (1)), and adding this isotropic magnetic contribution to the other spherically symmetric interaction energies. Although this approximation is valuable to estimate the field range needed for magnetic flocculation [6], it cannot be expected to give quantitatively correct results for the flocculation rate. 
Parker and co-workers [7] have proposed a different approach, based on classical mechanics rather than the usual flocculation theories. They construct a force-balance equation which incorporates all interparticle forces and the Stokes drag, and calculate the trajectory followed by a particle that flocculates with a fixed central particle, for many different initial positions of the moving particle. In this way they determine so-called isochronal surfaces, formed by all initial positions of the moving particle that lead to flocculation after the same elapsed time. Assuming that the initial positions all have equal probability, the total amount of flocculated particles at time $t$ is then proportional to the volume that is enclosed by the corresponding isochronal surface. Although this approach does account for the angular dependence of the magnetic interaction, it cannot give a complete description. The model does not contain terms depending on the thermal energy $k_{\mathrm{B}} T$ (since Brownian motion is not accounted for), and therefore it does not reproduce the well established results of Fuchs when the field is turned off. The natural way to describe magnetic flocculation kinetics is to recalculate the Fuchs model, while accounting for the rangetic interaction from the beginning. Wilhelm [8] has followed this approach for the magnetic flocculation of particles with permanent magnetic moments. The starting equations of his calculations of the initial flocculation rate are essentially the same as in our treatment of paramagnetic and diamagnetic particles that will be discussed below, but he has chosen a different mathematical approach to solve them.

\section{Flocculation in the presence of interparticle interactions: Fuchs' approach}

The theory of flocculation in the presence of interparticle interactions (often called the theory of slow flocculation, because one usually considers repulsive interactions which slow down the flocculation process) is based on an approach by Fuchs [5], originally developed for the flocculation of charged particles in air. In this model a monodisperse colloid of spherical particles with diameter $d$ in a liquid is considered. The total interaction energy for the interaction between two such particles is denoted as $V(\boldsymbol{r})$, where $\boldsymbol{r}$ is the vector connecting the particle centres. In the DLVO theory, the total interaction between two particles is considered to be the sum of the van der Waals attraction, and the electric double layer interaction [1, 2]. The van der Waals interaction between two spherical particles of diameter $d$ is given by:

$$
V_{\mathrm{A}}=-\frac{A}{12}\left(\frac{1}{r_{\mathrm{d}}^{2}-1}+\frac{1}{r_{\mathrm{d}}^{2}}+2 \ln \frac{r_{\mathrm{d}}^{2}-1}{r_{\mathrm{d}}^{2}}\right)
$$

where $r_{\mathrm{d}}=r / d$ and the so-called Hamaker constant $A$ has a value of the order $10^{-20} \mathrm{~J}$. The expression for the electric double layer interaction depends on the ratio of the particle diameter and the so-called Debye length
$1 / k$, which gives a measure for the spatial extent of the diffuse countercharge layer that surrounds the particle. For relatively wide double layers $(\kappa d<1)$ the interaction energy is given by $[1,2]$ :

$$
V_{\mathrm{R}}=\pi \varepsilon d \psi_{0}^{2} \frac{\exp \left[-\kappa d\left(r^{\mathrm{d}}-1\right)\right]}{r_{\mathrm{d}}}
$$

where $\varepsilon$ is the dielectric constant of the liquid, and $\psi_{0}$ is the electric potential difference between the particle surface and the bulk liquid.

The initial stage of the flocculation process can be modelled by placing one particle at the origin of the coordinate system, and assuming that all other particles flocculate only with this central particle and not among themselves. Furthermore the flocculation centre is assumed to remain unchanged during the process, which means that the particles reaching it simply disappear [1-5]. The initial stage of the flocculation process is then governed by the following continuity equation:

$$
\frac{\partial n}{\partial t}=-\boldsymbol{\nabla} \cdot j
$$

where $n$ is the particle number density and $j$ the particle flux, given by:

$$
j=-D_{12}\left(\nabla n+n \nabla V / k_{\mathrm{B}} T\right) .
$$

Here $D_{12}$ is the relative diffusion coefficient, which is introduced to account for the Brownian motion of the central particle, and is often approximated as twice the Einstein diffusion coefficient $D$ for a single particle [ 1 . 2]. The boundary conditions for $n$ are:

$$
\begin{aligned}
& n(d)=0 \\
& n(x)=n_{0} .
\end{aligned}
$$

The flocculation rate is the number of particles that reach the surface $r=d$ per unit time, which is just the flux through this surface:

$$
\begin{aligned}
& j_{\text {central }}(d)=d^{2} \int_{0}^{2 \pi} \mathrm{d} \varphi \int_{0}^{\pi} \sin \theta \mathrm{d} \theta \\
& \times\left. D_{12}\left\{(\nabla n)_{r}+n\left(\nabla V / k_{\mathrm{B}} T\right)_{r}\right\}\right|_{r=d} .
\end{aligned}
$$

For spherically symmetric interactions this yields [1]:

$$
j_{\text {central }}(d)=\left.8 \pi d^{2} D\left(\frac{\partial n}{\partial r}+n \frac{\partial V / k_{\mathrm{B}} T}{\partial r}\right)\right|_{r=d} .
$$

A full, time-dependent solution of (4) is very difficult, and has not yet been reported in the literature. However, under the common circumstances in colloid chemistry the process reaches a steady state very rapidly, and one generally assumes that $\partial n / \partial t=0$. We will consider this point in more detail in the subsequent paper [9]. In the steady state the divergence of the flux is zero, and the total flux through any spherical surface of radius $r$ is a constant number $J$. For spherically symmetric interactions (5) can then be solved for $n$, using the boundary conditions (6). Assuming for the 
interaction energy that $V(x)=0$ and $V(d)=-\infty$, we obtain [1]:

$$
n(r)=\mathrm{e}^{-V / k_{\mathrm{B}} T}\left(n_{0}+\frac{J}{8 \pi D} \int_{x}^{r} \frac{\mathrm{e}^{V(x) / k_{\mathrm{B}} T}}{x^{2}} \mathrm{~d} x\right) .
$$

The initial flocculation rate is then $[1,2]$ :

$$
J=\frac{8 \pi D n_{0} d}{W}
$$

where $W$ is the so-called stability factor, which indicates how the interactions change the total flux compared to rapid flocculation [4], in which no repulsive forces are present to slow down the flocculation process ( $W=1$ ). In the model presented in this section $W$ is given by:

$$
W=\int_{1}^{\infty} \frac{\mathrm{e}^{V\left(r_{\mathrm{d}}\right) / k_{\mathrm{B}} T}}{r_{\mathrm{d}}^{2}} \mathrm{~d} r_{\mathrm{d}} .
$$

\section{Numerical calculation of the initial flocculation rate in magnetic flocculation}

In our further analysis we will restrict ourselves to the magnetic flocculation of isotropic paramagnetic and diamagnetic materials, in which the magnetic moments are induced by an applied magnetic field, and are either parallel or antiparallel to this field. In that case the magnetic interaction (and through that the total interaction) has a cylindrical symmetry around the field direction. The results can be rewritten to describe the magnetic flocculation of perfectly oriented permanent dipoles, through replacement of the induced magnetic moment $\frac{1}{6} \pi d^{3} \chi B / \mu_{0}$ by the permanent moment $m$ in the final results.

The starting point is again (5), but now the interaction energy $V$ includes the magnetic dipole-dipole interaction, and thus depends also on the angle $\theta$ between $\boldsymbol{r}$ and the applied magnetic field $\boldsymbol{B}$ (which defines the $z$ axis). We write the number density $n$ as:

$$
n(r, \theta)=n_{0} f(r, \theta) \mathrm{e}^{-\gamma(r, \theta) / 2 k_{\mathrm{B}} T}
$$

where $f(r, \theta)$ is a dimensionless function, to be determined below. The boundary conditions for $f$ follow from the corresponding conditions for $n$ (equation (6)):

$$
\begin{aligned}
& f(d, \theta)=0 \\
& f(x, \theta)=1 .
\end{aligned}
$$

Substitution of (12) in equations (4) and (5) results in a second-order differential equation for $f$ in the steady state $(\partial n / \partial t=0)$ :

$$
\Delta f-\Psi(r, \theta) f=0
$$

where $\Psi(r, \theta)$ is defined as:

$$
\Psi(r, \theta)=\left(\nabla V / k_{\mathrm{B}} T\right)^{2} / 4-\Delta V / 2 k_{\mathrm{B}} T .
$$

Equation (14) is a so-called self-adjoint equationt,

\footnotetext{
+ It should be noted that the factor $\frac{1}{2}$ in the argument of the exponential in equation (12) is essential to let the terms containing $\nabla f$ vanish, leading to this type of equation. In similar substitutions by several other authors e.g. Wilhelm [8], this factor is left out, and another mathematical approach is followed.
}

which has some convenient mathematical properties [10]. $f$ can be expanded as:

$$
f(r, \theta)=\sum_{m} f_{2 m}(r) P_{2 m}(u)
$$

where $u=\cos \theta$, and $P_{2 m}(u)$ is the Legendre polynomial of order $2 \mathrm{~m}$. The expansion contains only even orders because of the symmetry of the interaction. The functions $f_{2 m}(r)$ are not specified by an analytical expression, and must be determined numerically.

Substitution of this series expansion in (14) yields:

$$
\sum_{m} P_{2 m} \Delta_{r} f_{2 m}+\sum_{m} f_{2 m} \Delta_{\theta} P_{2 m}=\sum_{m} f_{2 m} P_{2 m} \Psi
$$

where $\Delta_{r}$ and $\Delta_{\theta}$ denote the parts of the Laplace operator $\Delta$ that contain derivatives with respect to $r$ and $\theta$ respectively. For Legendre polynomials we have [10]:

$$
\Delta_{\theta} P_{l}=-\frac{l(l+1)}{r^{2}} P_{l}
$$

We now substitute this in (17), multiply both sides with the Legendre polynomial $P_{2 l}(u)$, and integrate over $u$ from -1 to 1 :

$$
\begin{aligned}
& \sum_{m}\left(\Delta_{r} f_{2 m}-f_{2 m} \frac{2 m(2 m+1)}{r^{2}}\right) \int_{-1}^{1} \mathrm{~d} u P_{2 l} P_{2 m} \\
& \quad=\sum_{m} f_{2 m} \int_{-1}^{1} \mathrm{~d} u P_{2 l} \Psi P_{2 m} .
\end{aligned}
$$

Using the orthogonality relation for the Legendre polynomials [11] this becomes:

$$
\frac{2}{4 l+1} \Delta_{r} f_{2 l}+\frac{8 l}{r^{2}} f_{2 l}=\sum_{m} f_{2 m} \Psi_{2 l .2 m}
$$

where:

$$
\Psi_{2 l, 2 m}=\int_{-1}^{1} \mathrm{~d} u P_{2 l} \Psi P_{2 m} .
$$

$\Psi_{2 l .2 m}$ can be calculated analytically for a purely magnetic interaction, or numerically for the general case.

Equation (20) represents a set of coupled, secondorder differential equations. The boundary conditions for the functions $f_{2 l}$ follow from the boundary conditions of $f$. At $r=d$ we get:

$$
f_{2 l}(d)=0 \quad \text { for all } l .
$$

For $r$ tending to infinity, we want the isotropic solution to be the only one left. The condition $f(\infty)=1$ is then satisfied with:

$$
\begin{aligned}
& f_{0}(x)=1 \\
& f_{2 l}(x)=0 \quad \text { for } l \neq 0 .
\end{aligned}
$$

In practice one can now take a finite number of terms, high enough to give satisfactory accuracy, and solve the set of coupled differential equations numerically. In our calculations the van der Waals interaction between spheres of diameter $d$ is used in its usual form (equation (2)), choosing a value of $7 \times 10^{-211} \mathrm{~J}$ for the 
Hamaker constant $A$. The double-layer repulsion and the magnetic interaction are rewritten in terms of some convenient parameters, in order to make the results as general as possible. The double-layer interaction is represented as:

$$
\frac{V_{\mathrm{R}}}{k_{\mathrm{B}} T}=V_{\mathrm{R} 0} \frac{\mathrm{e}^{-\kappa d r_{\mathrm{d}}}}{r_{\mathrm{d}}}
$$

where $V_{\mathrm{R} 0}$ is given by:

$$
V_{\mathrm{R} 0}=\frac{\pi \varepsilon_{0} \varepsilon_{r} d \psi_{0}^{2}}{k_{\mathrm{B}} T} \mathrm{e}^{\kappa d}
$$

The magnetic interaction, equation (1), can be written as:

$$
\frac{V_{\mathrm{M}}}{k_{\mathrm{B}} T}=-\frac{2}{\sqrt{3}} \frac{\beta^{2}}{r_{\mathrm{d}}^{3}} P_{2}(u)
$$

where the dimensionless interparticle distance is $r_{\mathrm{d}}=$ $r / d$, and the dimensionless parameter $\beta$ is given by:

$$
\begin{aligned}
\beta & =\chi B d^{3 / 2} \sqrt{\frac{\sqrt{3} \pi}{144 \mu_{0} k_{\mathrm{B}} T}} \\
& =2728 \chi B d^{3 / 2} \quad d \text { in } \mu \mathrm{m} .
\end{aligned}
$$

Details of the numerical procedure will be published elsewhere [12]. Here we will just mention the most important results. To start with, we note that the convergence of the series equation (16) turns out to be quite rapid. For an accuracy of 14 digits, no more than 8 terms are necessary at low-field values. For high fields the main contribution turns out to result from the isotropic $l=0$ term, the other ones giving only minor corrections. Note however, that this conclusion does not justify Svoboda's isotropic approximation [6]. In his work the total interaction is spherically symmetric, and produces a spherically symmetric particle flux. In our case the flux still has an angle dependence, through the factor $\exp \left(-V(r, \theta) / 2 k_{\mathrm{B}} T\right)$ in equation (12).

Representative results are given in figure 1 for $\kappa d=$ 1 , in terms of the stability factor $W$ as a function of $\beta$. The different curves correspond to different values of $V_{\mathrm{R} 0}$ (equation (25)), which determines the zero-field stability. For small values of $\beta$ the effect of the magnetic interaction is negligible, as can be expected when $\left|V_{\mathrm{M}} / k_{\mathrm{B}} T\right| \ll 1$. For large values of $\beta$ however, all curves come together. In that region the magnetic interaction is dominant, and $W$ depends on $\beta$ as $W=$ $3.014678(5) \times \beta^{-2 / 3}$. Experimentally we then expect to see that the relation between the flocculation rate $(\sim 1 /$ $W$, equation (10)) and the magnetic field $B$ is a $B^{2 / 3}$ power law.

\section{Principle of the experiments on magnetic flocculation kinetics}

In our experiments we study the time-dependence of the extinction, for light that is incident along the magnetic field. When multiple scattering is negligible, the

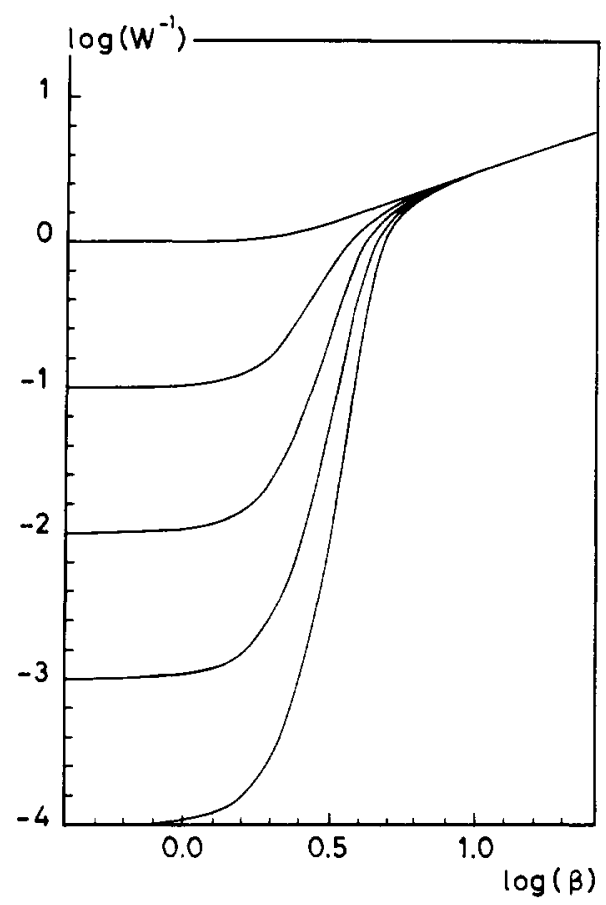

Figure 1. Calculated dependence of the stability factor $W$ on the magnetic interaction parameter $\beta$ for $\kappa d=1$. At high fields this relation is a $\beta^{-2 / 3}$ power law (after [12]). The various curves correspond to different values of the zerofield stability, determined by $V_{R O}$.

extinction of light by a colloid is given by the LambertBeer law [13]:

$$
I(t)=I_{0} \exp \left(-L \sum_{i} n_{i}(t) C_{i}\right)
$$

where $I$ is the transmitted intensity, $I_{0}$ the incident intensity, $L$ the distance the light travels through the colloid, $n_{i}(t)$ the (time-dependent) number density of scatterers of type $i$, and $C_{i}$ is the corresponding extinction coefficient of one such scatterer.

In the initial stage of the process only single particles and doublets will be present, and we have:

$$
\ln \frac{I(t)}{I_{0}}=-L\left[C_{1} n_{1}(t)+C_{2} n_{2}(t)\right] .
$$

Furthermore $n_{2}(t)=\left(n_{1}(0)-n_{1}(t)\right) / 2$, since the disappearing single particles are aggregating into doublets. This yields:

$$
\ln I(t)=K_{1}+K_{2} n_{1}(t)
$$

where:

$$
\begin{aligned}
& K_{1}=\ln I_{0}-L C_{2} n_{1}(0) / 2 \\
& K_{2}=-L\left(C_{1}-C_{2} / 2\right) .
\end{aligned}
$$

It should be noted that $K_{2}$ can be positive and negative. For particles in the geometrical optics limit [13], an oriented doublet at end-on illumination has the same geometrical shadow as a single particle. Then $C_{1}=C_{2}$ and $K_{2}<0$. For smaller particles however, it is well possible that the extinction coefficient of a doublet is larger than that of two single particles, and then $K_{2}$ 
can be positive. The transition rate for the singletdoublet transition for $t \rightarrow 0$ is found by differentiation of equation (30) with respect to time:

$$
\begin{aligned}
\left(\frac{\mathrm{d} n_{1}}{\mathrm{~d} t}\right)_{t \rightarrow 0} & =\frac{1}{K_{2}}\left(\frac{\mathrm{d}}{\mathrm{d} t} \ln I(t)\right)_{t \rightarrow 0} \\
& =\frac{1}{K_{2} I(0)}\left(\frac{\mathrm{d} I}{\mathrm{~d} t}\right)_{t \rightarrow 0}
\end{aligned}
$$

In the initial stage of the process the flocculation rate calculated in section 3 (figure 1 and equation (10)) thus is directly proportional to the initial slope of the experimental $I(t)$ versus $t$ curve. In the high-field limit, this slope $(\mathrm{d} I / \mathrm{d} t)_{t \rightarrow 0}$ should vary according to a $B^{2 / 3}$ power law.

\section{Experimental set-up and samples}

In the extinction measurements we want to be able to detect small changes in the intensity of the transmitted light, which is itself a fairly large signal. We have accomplished this by sensitive measurement of the difference between the actual transmission and a reference signal representing the transmission in zero field. To this end, our set-up contains two identical light emitting diodes (LED) with maximum intensity at a wavelength of $890 \mathrm{~nm}$. The light of one LED is coupled into a glassfibre light guide that leads to the sample cell. A second light guide leads the transmitted light to the detector, a photo-transistor circuit. The light of the second LED is focused directly onto this detector. The two LEDs are driven alternately by a blockwave-generator at $333 \mathrm{~Hz}$, and so the $\mathrm{AC}$ signal from the detector is proportional to the difference between the intensity of the light transmitted through the sample and the (adjustable) intensity of the reference signal. This AC signal is measured phase-sensitively. Since the two LEDS are driven by the same oscillator, and a single detector is used, drifts in the oscillator output and the detector sensitivity are eliminated to a large extent: we have obtained a residual drift of about $10^{-4}$ parts of the actual transmission per hour. The mechanical system is shown schematically in figure 2 . The sample is kept outside the magnet until the field has reached the desired value (which can take up to about $20 \mathrm{~s}$ for the highest field of $15 \mathrm{~T}$ ). Then the sample is moved to the magnet centre in a fraction of a second. In this way the magnetic interaction can be considered as switched on instantaneously, up to the highest fields used. The field at the initial position of the sample (about $50 \mathrm{~cm}$ above the magnet centre) is only $1.5 \%$ of the value at the centre, and is negligible for all but the most unstable colloids. A microswitch indicates the arrival of the sample at the magnet centre, by leaving a mark on the paper of the $x-t$ recorder.

The colloids used in these experiments have been prepared by dispersion of ball-milled $\mathrm{Mn}_{2} \mathrm{O}_{3}$ powder in aqueous solutions of various amounts of

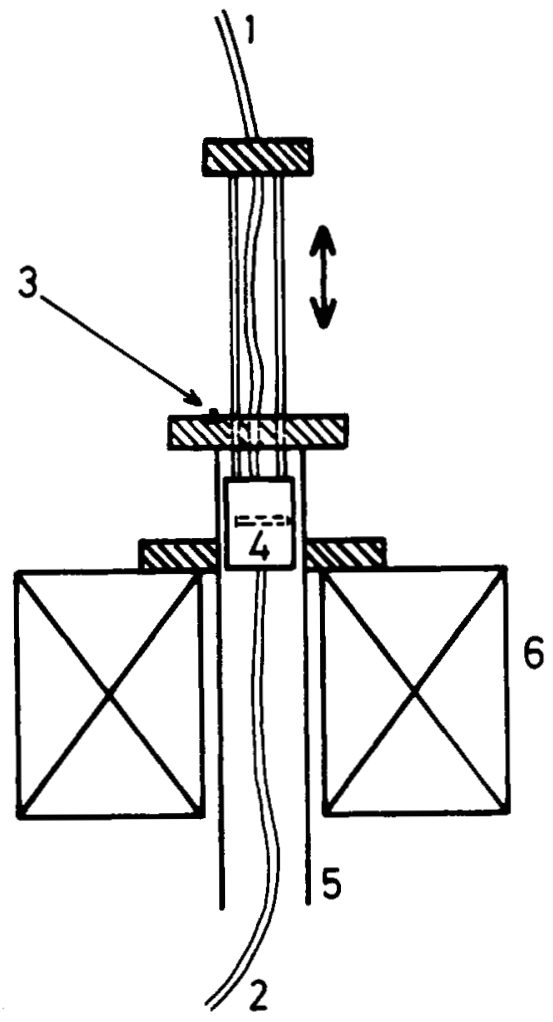

Figure 2. Mechanical part of the experimental set-up: 1, light guide from light source; 2 , light guide to detector; 3 , microswitch; 4, movable sample holder; 5 , guiding tube; 6 , Bitter magnet.

$\mathrm{Na}_{4} \mathrm{P}_{2} \mathrm{O}_{7} \cdot 7 \mathrm{H}_{2} \mathrm{O}$ (typically 1 or 2 grams per litre). They were allowed to age for some time (up to three months) before use, to let any remaining large particles and aggregates settle out. The size distribution in such colloids is rather broad. In principle, filtration through membranes of well defined pore size could have been used to narrow this distribution, but it is very tedious to prepare sufficient quantities of colloid of a high enough concentration this way. In these experiments we have made a distinction only between coarse-particle colloids [9], in which the constituent particles are microscopically observable, and fine-particle colloids (used in this work) that are too small for such an observation. The zero-field stability of most of the samples used in this work has been reduced somewhat by the addition of $\mathrm{KCl}$, so that the transition to dominance of the magnetic interaction occurs in the range of fields available in a $15 \mathrm{~T}$ Bitter magnet.

\section{Experimental results and discussion}

A typical example of the transmitted intensity as a function of time is shown in figure 3 . In this example, and in all other measurements on dilute fine-particle colloids, the initial part of the transmission curve shows 


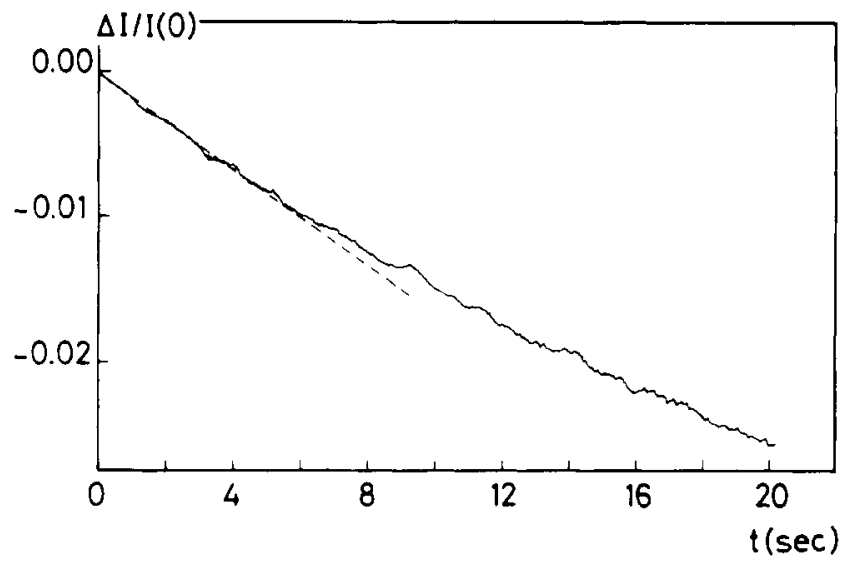

Figure 3. Typical example of the relative change in the transmitted intensity as a function of time, for a fine-particle $\mathrm{Mn}_{2} \mathrm{O}_{3}$ colloid. The applied field is $12 \mathrm{~T}$. The broken line is a guide to the eye. Its slope is the experimental quantity of interest.

a linear dependence on time within the noise level, allowing an unambiguous determination of the initial slope. Figure 4 shows an example of the field-dependence of this slope. We see that the theoretically predicted dependence (see figure 1) is indeed nicely reproduced. In particular the $B^{2 / 3}$ power law is clearly present at high fields. Estimates for the particle size can be obtained by comparison of figures 1 and 4 . In figure 1 the steepest part of the curve is located at $\log \beta \approx 0.45$, and the transition to the $\beta^{2 / 3}$ relation occurs at $\log \beta \simeq 0.85$. In figure 4 these points lie at $\beta / d^{3 / 2} \simeq 40$ and $\beta / d^{3 / 2} \simeq 100$, both leading to a diameter of $0.17 \mu \mathrm{m}$ (in agreement with the very slow sedimentation and with the fact that these particles are not visible under an optical microscope).

Which part of the theoretical curve can be observed experimentally, is determined by the stability conditions of the sample. For samples that are more stable than the ones used to obtain figure 4 , the transition to the $B^{2 / 3}$ power law occurs above $15 \mathrm{~T}$, and is not experimentally accessible with the Bitter magnet that was used here. In figure 5 an example of such a measurement is shown. The colloid used is essentially the same as in figure 4 , except that no $\mathrm{KCl}$ has been added.

Finally we want to make some comments on the earlier treatments by Svoboda [6] and Wilhelm [8]. First we want to make a comparison between our results and Svoboda's isotropic approximation of the same theoretical problem, for the case of dominant magnetic interaction. The stability factor in Svoboda's approach then reads:

$$
W=\beta^{-2 / 3} \int_{0}^{\beta^{2 / 3}} \mathrm{~d} z \mathrm{e}^{-2 z^{3} / \sqrt{ }}
$$

where the variable $z=\beta^{2 / 3} / r_{\mathrm{d}}$ has been introduced into equation (11). For large $\beta$ the integral tends to a value of 0.8517 and we get $W=0.8517 \beta^{-2 / 3}$, while the exact

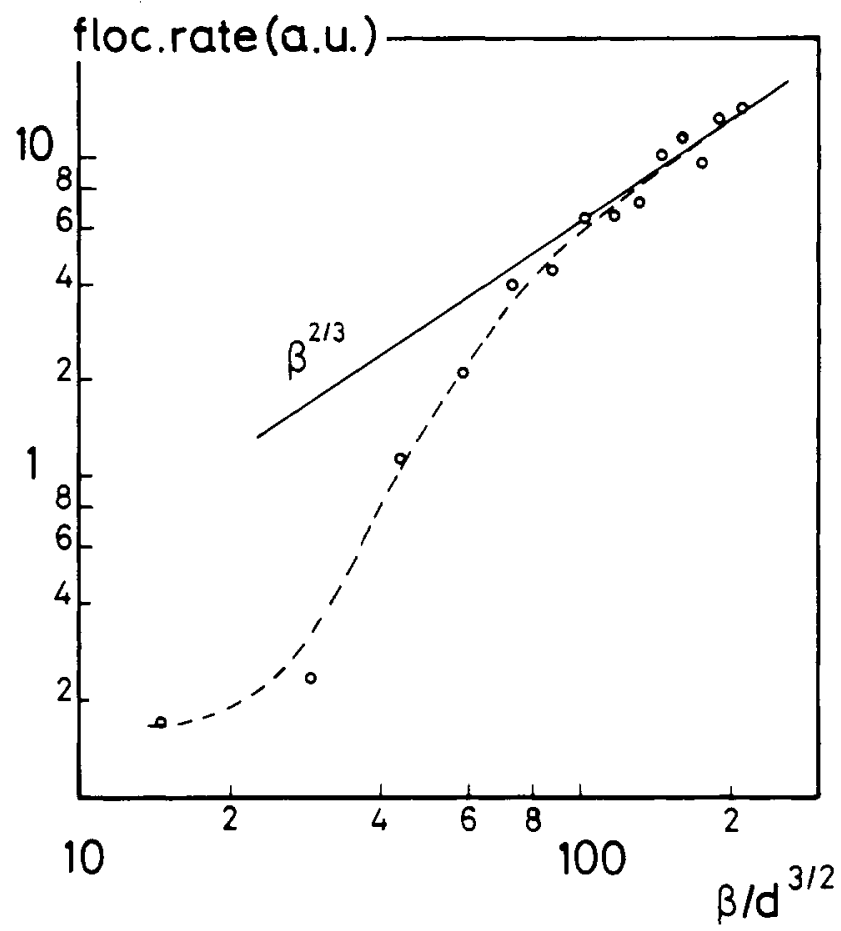

Figure 4. Flocculation rate versus the parameter $\beta / d^{3 / 2}$, which is equal to $14.5 B$ for $B$ in Tesla and $d$ in $\mu \mathrm{m}$ (see equation (27)). The broken curve is a guide to the eye. The full line indicates a $\beta^{2 / 3}$ power law.

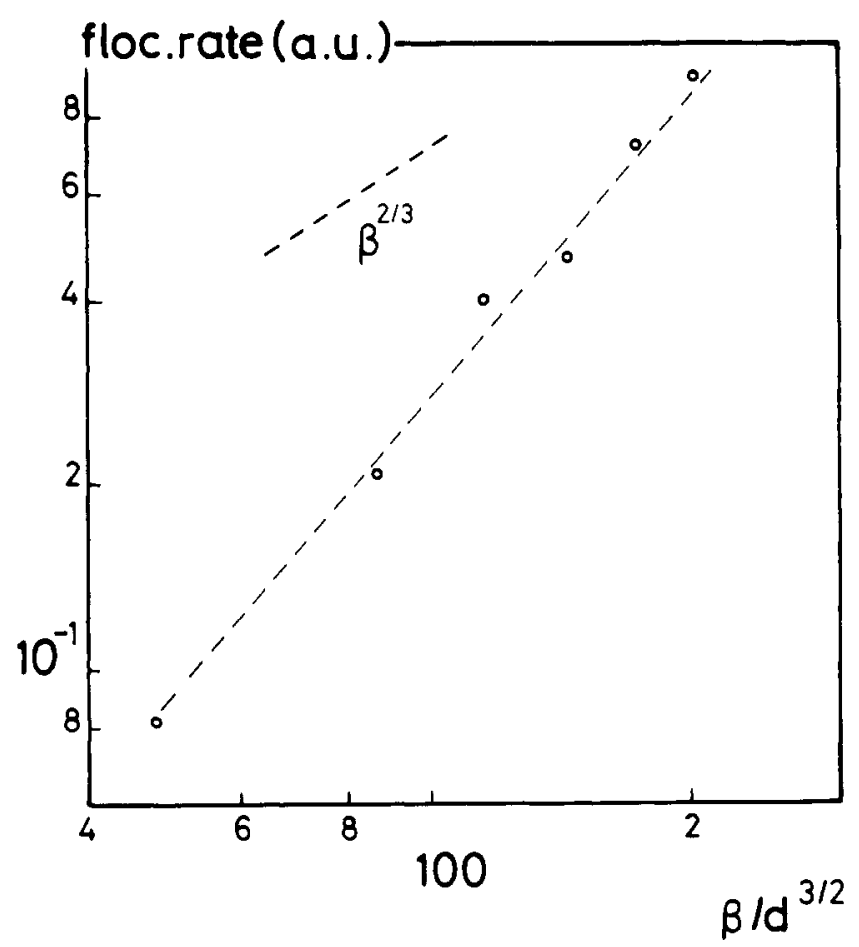

Figure 5. Flocculation rate versus $\beta / d^{32}$ for a colloid that is more stable (containing no $\mathrm{KCl}$ ) than the one used to obtain figure 4 . The $\beta^{2 / 3}$ power law is not reached in the available field range (up to $15 \mathrm{~T}$ ). 
results give $W=3.014678(5) \times \beta^{-2 / 3}$. So, although the $\beta^{-2 / 3}$-dependence is correctly predicted, Svoboda overestimates the flocculation rate at high fields by a factor 3.5. Furthermore the importance of the magnetic interaction at low fields is too large in his calculations. For $\beta=1$ for instance, figure 1 shows that the influence of the magnetic interaction is negligible for the curve with $W=1$ for zero field. Equation (34) however gives $W \simeq$ 0.78 .

Wilhelm [8] bases his treatment of the flocculation of identical particles with permanent magnetic moments on equations (4) and (5), as is done in our approach. Using the substitution $n(r, \theta)=N(r, \theta)$ $\exp \left(-V / k_{\mathrm{B}} T\right)$ he finds for the steady state:

$$
\boldsymbol{\nabla} \cdot\left(\mathrm{e}^{-V / k_{\mathrm{B}} T} \boldsymbol{\nabla} N\right)=0
$$

where the particle flux is given by:

$$
j=-D_{12} \mathrm{e}^{-V / k_{\mathrm{B}} T} \nabla N .
$$

Now he introduces a so-called stream function $S(r, \theta)$ through:

$$
j=-D_{12}\left(\frac{\partial S / \partial \theta}{r^{2} \sin \theta} \hat{\boldsymbol{r}}-\frac{\partial S / \partial r}{r \sin \theta} \hat{\boldsymbol{\theta}}\right)
$$

thus automatically satisfying $\boldsymbol{\nabla} \cdot \boldsymbol{j}=0$. As an analytical expression for $S$, Wilhelm proposes:

$$
S(r, \theta)=-n_{0} d \cos \theta \mathrm{e}^{-V / k_{\mathrm{B}} T} .
$$

For this choice, von Smoluchowski's model [4] is reproduced for $V=0$. However, it does not give correct results for a spherically symmetric interaction $V(r)$.

Substitution of such an interaction in equation (37) leads to:

$j=-\frac{n_{0} D_{12} d \mathrm{e}^{-V / k_{\mathrm{B}} T}}{r}\left(\frac{1}{r} \hat{\boldsymbol{r}}+\frac{\cos \theta}{\sin \theta} \frac{\partial V / k_{\mathrm{B}} T}{\partial r} \hat{\boldsymbol{\theta}}\right)$

in contradiction with the fact that a spherically symmetric interaction should lead to a spherically symmetric particle flux.

Due to this inadequate choice of the stream function, Wilhelm is led to some counter-intuitive results. In his calculations of the initial flocculation rate for particles with a permanent magnetic moment, he finds for instance that this rate is smaller in the presence of a magnetic field than in zero field, and claims that this is experimentally confirmed in observations of the decay of a magnetic aerosol (and used in practice in the fabrication of magnetic aerosols). This statement however, is in contradiction with flocculation experiments on ferromagnetic maghemite $\left(\gamma-\mathrm{Fe}_{2} \mathrm{O}_{3}\right)$ colloids that we have performed. In these experiments the magnetic field clearly has a destabilizing effect, and the flocculation process is observed to occur faster when the field is increased. In figure 6 the initial flocculation rate for such a colloid is shown as a function of the magnetic field. This rate has been determined from the

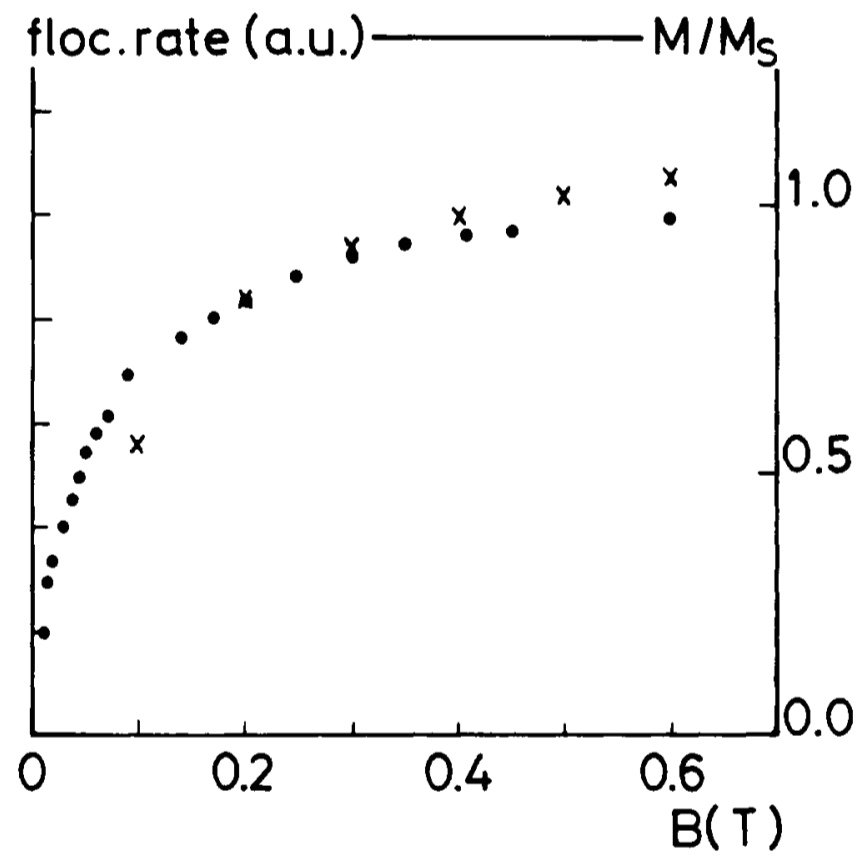

Figure 6. Flocculation rate (crosses) and normalized magnetization (full circles) for an electrostatically stabilized $\gamma-\mathrm{Fe}_{2} \mathrm{O}_{3}$ colloid as a function of the applied magnetic field. The vertical scale for the flocculation rate has been chosen so as to illustrate best the coincidence with the magnetization curve.

initial slope of the transmission curve, as described earlier, only this time the experiment was performed in a Varian electromagnet, in a geometry where the field is perpendicular to the lightpath. We see that the flocculation rate increases with the magnetic field initially, and levels off when the magnetic moments are almost perfectly oriented. Indeed the flocculation rate has essentially the same field dependence as the magnetization of the colloid, which was measured with a vibrating sample magnetometer and is shown also in figure 6 . The magnetic stabilization of magnetic aerosols, mentioned by Wilhelm, has another explanation in our opinion. We believe that these observations are made beyond the initial stage of the flocculation process, in the presence of larger aggregates. In zero field these aggregates are more or less spherical, and new particles can attach to them from all sides. In a strong applied field however, the aggregates will be elongated and oriented, and the magnetic moments within an aggregate will be oriented along the external field. The magnetic force on an approaching particle will then be such that attachment along a non-axial direction is suppressed, and the growth of the aggregate occurs mainly at the end-points. Then the flocculation rate may be lower in the presence of an applied field than in zero field, provided that the flocculation process has proceeded beyond the initial stage. 


\section{Conclusions}

A numerical approach has been presented to incorporate cylindrically symmetric interactions into the established flocculation model of Fuchs. Applied to magnetic flocculation of non-ferromagnetic particles, this approach gives the dependence of the colloid's stability factor on the magnetic field. In particular a $B^{-2 / 3}$ power law dependence is predicted for high fields, where the magnetic interaction is dominant. Both the shape of the calculated curves and the $B^{-2 / 3}$ power law at high fields were verified experimentally for magnetic flocculation of aqueous $\mathrm{Mn}_{2} \mathrm{O}_{3}$ colloids, containing particles with a diameter of about $0.2 \mu \mathrm{m}$.

\section{Acknowledgments}

Part of this work has been supported by the Stichting voor Fundamenteel Onderzoek der Materie (FOM) and the Stichting voor de Technische Wetenschappen (STW), with financial support from the Nederlandse Organisatie voor Wetenschappelijk Onderzoek (NWO).

\section{References}

[1] Kruyt H R (ed) 1952 Colloid Science part I (Amsterdam: Elsevier)

[2] Sonntag H and Strenge K 1987 Coagulation Kinetics and Structure Formation (New York: Plenum)

[3] Friedlander S K 1977 Smoke, Dust and Haze (New York: Wiley)

[4] von Smoluchowski M 1917 Z. Phys. Chem. 92129

[5] Fuchs N A 1934 Z. Phys. 89736

[6] Svoboda J 1982 IEEE Trans. Magn. MAG-18 796

[7] Parker M R, van Kleef R P A R, Myron H W and Wyder P 1984 J. Colloid Interface Sci. 101314

[8] Wilhelm H E 1986 Phys. Fluids 291441

[9] Janssen J J M, Baltussen J J M, van Gelder A P and Perenboom J A A J 1990 J. Phys. D: Appl. Phys. 23

[10] Arfken G 1985 Mathematical Methods for Physicists (New York: Academic) ch 9

[11] Arfken G 1985 Mathematical Methods for Physicists (New York: Academic) ch 12

[12] van Gelder A P 1990 to be published

[13] van de Hulst H C 1957 Light Scattering by Small Particles (New York: Wiley) 